\title{
New experimental results supporting the hypothesis of the excitation of collective modes in the ${ }^{40} \mathrm{Ca}+{ }^{40} \mathrm{Ca}$ reaction
}

\author{
H. Tricoire, P. Colombani, C. Gerschel, D. Paya (*), N. Perrin, L. Valentin, N. Frascaria, J. P. Garron \\ and C. Stéphan \\ Institut de Physique Nucléaire, B.P. no 1, 91406 Orsay, France
}

(Reçu le 8 février 1979, accepté le 5 mars 1979)

\begin{abstract}
Résumé. - Dans la réaction $\mathrm{Ca}+\mathrm{Ca}$ à $400 \mathrm{MeV}$, nous avons mesuré la multiplicité $\gamma$ en fonction de l'énergie des fragments. Des paliers de multiplicité ont été mis en évidence dans la région incomplètement relaxée en énergie. Leur dépendance angulaire et leur énergie sont la signature de processus directs et suggèrent qu'ils pourraient être liés à l'excitation de modes collectifs de haute énergie prévue théoriquement. Une discussion des valeurs de $l$ les plus probables pour ces excitations est présentée.
\end{abstract}

\begin{abstract}
Steps in the $\gamma$-ray multiplicity as function of the excitation energy have been observed in coincidence with the incompletely relaxed part of the energy spectra of fragments emitted in the reaction ${ }^{40} \mathrm{Ca}+{ }^{40} \mathrm{Ca}$ at $400 \mathrm{MeV}$. Excitation energies and angular dependence of these variations suggest that they are correlated with the excitation of high energy collective modes through a rather direct process in the early stages of the reaction. $l$-values of this excitation mode are discussed.
\end{abstract}

Recent studies of fragments emitted in the reactions ${ }^{40} \mathrm{Ca}+{ }^{40} \mathrm{Ca}$ at $284 \mathrm{MeV}$ and ${ }^{63} \mathrm{Cu}+{ }^{63} \mathrm{Cu}$ at $450 \mathrm{MeV}$ [1] have shown the existence of some structures, unresolved up to now, in the incompletely relaxed component of the energy spectrum. These structures (bumps) were observed for fragments of $Z$ and $N$ not too different from the projectile. In the ${ }^{40} \mathrm{Ca}+{ }^{40} \mathrm{Ca}$. system, they were found at a total excitation energy of about $50 \mathrm{MeV}$, and it was shown that, for a given $Z$ of the detected fragment, this energy did not change appreciably either with the C.M. angle or with the isotope considered. The angular distribution of these structures was found to be strongly forward peaked, which, together with the sharp $(Z, N)$ distribution, implies a rather short interaction time between the ions. A tentative explanation for these structures seemed to be the excitation of collective modes in the early stage of the reaction, as predicted by Broglia, Dasso and Winther [2]. Confirmation of the structure at $50 \mathrm{MeV}$ as well as some indication for another bump at $80 \mathrm{MeV}$ in some isotopes has been obtained more recently in Orsay [3] for the same system studied at $400 \mathrm{MeV}$.

(*) Permanent address : C.E.N. Saclay, 91190 Gif sur Yvette, France.
In this letter we present a study of the $\gamma$-deexcitation of the emitted fragments in the reaction ${ }^{40} \mathrm{Ca}+{ }^{40} \mathrm{Ca}$ at $400 \mathrm{MeV}$. At this higher energy, a larger energy gap between the quasi-elastic component and the completely relaxed events allows a detailed investigation of the incompletely relaxed component. The $\gamma$-multiplicity of the fragment deexcitation has been measured in all three regions and some evidence for sudden variations of this quantity in the incompletely relaxed region has been found.

The $400 \mathrm{MeV}{ }^{40} \mathrm{Ca}$ beam was accelerated at the Orsay ALICE facility. $450 \mu \mathrm{g} / \mathrm{cm}^{2}{ }^{40} \mathrm{Ca}$ targets on a $10 \mu \mathrm{g} / \mathrm{cm}^{2}{ }^{12} \mathrm{C}$ backing were used. Contribution of reactions due to the backing was unimportant in the region of interest but was however subtracted by comparing the results with those obtained with a ${ }^{12} \mathrm{C}$ target. $Z$ identification of the reaction products was made by means of a solid state $E(200 \mu)-\Delta E(8 \mu)$ telescope, which provided an excellent separation between the different $Z$ up to $Z=28$. Four measurements were performed at $9.5^{\circ}, 10.5^{\circ}, 13^{\circ}, 25^{\circ}$ in the laboratory (the grazing angle is at $\simeq 10^{\circ}$ ). $\gamma$-rays were detected in coincidence with the heavy fragments in three $\mathrm{NaI}$ detectors $18 \mathrm{~cm}$ away from the target. Two of them were in the reaction plane, and the third one was in the perpendicular direction. Both single $E . \Delta E$ events and four-dimensional correlated 
events $E, \Delta E, E_{\gamma}, T$ (where $T$ is the time difference between $\gamma$-ray and fragment detection) were recorded on tapes and analysed off-line. The comparison of the time spectra at the three $\gamma$ detector angles show that the neutron contribution to the $\gamma$-spectra was unimportant.

Energy spectra and associated $\gamma$-multiplicities for $Z=18$ and $Z=19$ at $9.5^{\circ}, 10.5^{\circ}, 13^{\circ}$ are shown in figures 1 and 2 . For these $Z$ values, as well as for $Z=20$, the energy spectra exhibit all three components (quasi-elastic, incompletely relaxed, completely relaxed). However, results for $Z=20$ are less significant since the huge tail of the elastic peak at $9.5^{\circ}$ and $10.5^{\circ}$ spreads up to relatively high excitation energies and is expected to lower the multiplicity values. On part $a$ ) of these figures, the laboratory kinetic energy spectra at $9.5^{\circ}, 10.5^{\circ}$ and $13^{\circ}$ are displayed. The total excitation energy, calculated taking in account the evaporation of particles [4] is also indicated. Since we had no $A$ identification, all isotopes of a given element are summed in these spectra. Because of differences in $Q$-values and small differences in the position of the structures for different isotopes, the bumps are somewhat washed out and do not show up clearly. However, the incompletely relaxed part of the energy spectrum behaves quite differently at the various laboratory angles, showing some evidence for a broad bump at the most forward angles. Moreover, the experiment of ref. [3] leaves no doubt about their existence.

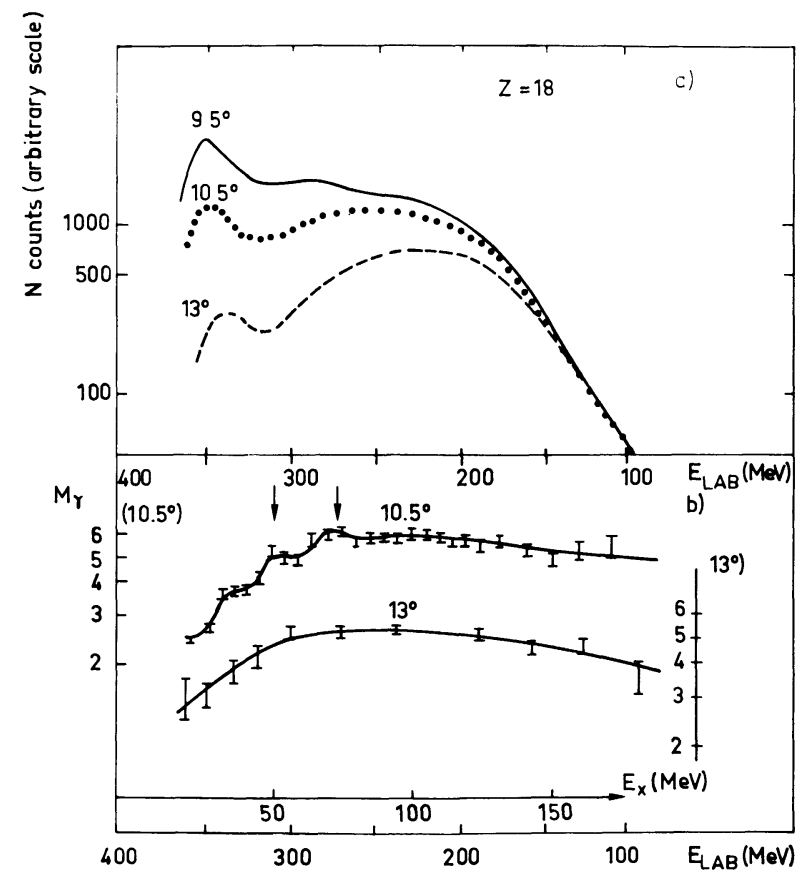

Fig. 1. - Part a) Kinetic energy spectra (lab.) of the $Z=18$ fragment at $9.5^{\circ}, 10.5^{\circ}, 13^{\circ}$. The relative scaling of the number of counts is arbitrary. Part $b$ ) Multiplicity spectra at $10.5^{\circ}$ and $13^{\circ}$ (lab.). The arrows show the position of the structures observed in ref. [3] in ${ }^{37} \mathrm{Ar}$ isotopes. Only statistical uncertainties are indicated. The total excitation energy is also indicated.

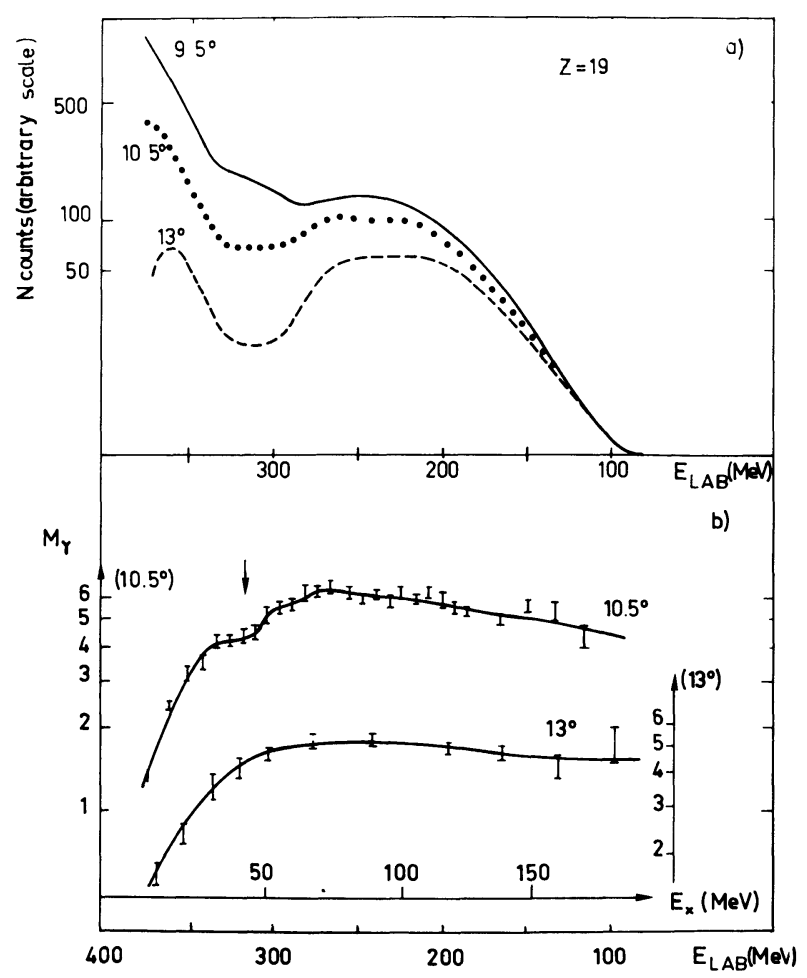

Fig. 2. - Same as figure 1 for $Z=19$. The arrow shows the position of the structure observed in ${ }^{40} \mathrm{~K}$ in ref. [3].

On part $b$ ) of these two figures, the corresponding multiplicities (deduced from the sum of events in the three $\mathrm{NaI}$ ) are shown at $10.5^{\circ}$ and $13^{\circ}$. Statistical uncertainties were too large at $9.5^{\circ}$ to allow an accurate analysis. Even at $13^{\circ}$ statistics are not as good as at $10.5^{\circ}$ but nevertheless the comparison between the two spectra is instructive. The main features of these spectra are the following :

- Increase of the multiplicity from the quasielastic to the completely relaxed region corresponding to an increase of the contact time and as a consequence to an increase in the transferred angular momentum.

- The maximum value of the multiplicity is obtained at a total excitation energy of about $100 \mathrm{MeV}$ and is not very high $\left(M_{\gamma} \simeq 6\right)$, that is $M_{\gamma} \simeq 3$ for each fragment. Since only a small number of $\gamma$ 's is emitted per event (the $\gamma$ energy spectra associated with these $Z$ values do not exhibit any kind of yrast bump), the angular momentum carried away by $\gamma$-deexcitation is very small compared to the total transferred angular momentum $(\simeq 30 \hbar$ given with $\langle l\rangle=100 \hbar$ by $\frac{2}{7}\langle l\rangle$, evaluation valid as the system is symmetric, for both the rolling and the sticking hypotheses). It is clear that, due to the high excitation energy of the system, a large number of particles are emitted in this region, carrying away with them most of the angular momentum. We will come back to this point later.

- Above about $100 \mathrm{MeV}$ total excitation energy, there is a slow decrease of the multiplicity which may 


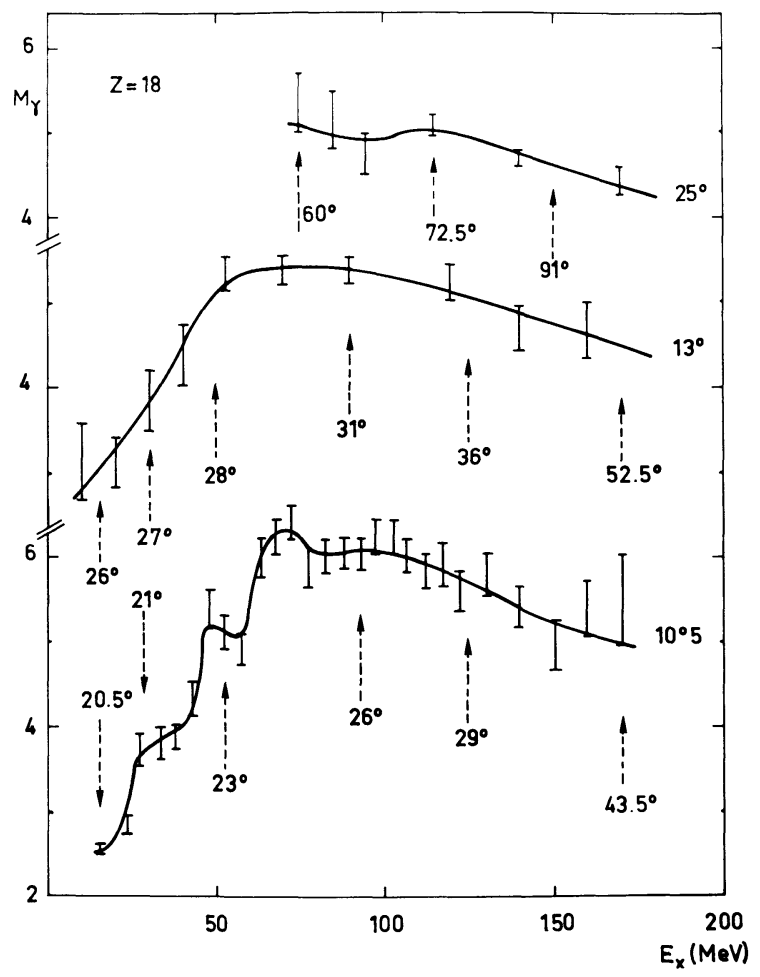

Fig. 3. - $\gamma$-ray multiplicity spectra at $10.5^{\circ}, 13^{\circ}, 25^{\circ}$ (lab.) as function of the total excitation energy, showing the variation of the centre of mass angle with the laboratory angle and the excitation energy.

be explained by the increasing number of evaporated particles and by the variation of the centre of mass angle with the excitation energy (see Fig. 3 at $10.5^{\circ}$, $13^{\circ}, 25^{\circ}$ (lab.) for $Z=18$ ). One should note that, in the incompletely relaxed region, the centre of mass angle is almost constant for a given laboratory angle, thus allowing a direct comparison of the multiplicity values.

- The most interesting feature of the multiplicity spectra precisely occurs in the incompletely relaxed region : at $10.5^{\circ}$, the spectra exhibit steps centred at a total excitation energies of 45 and $55 \mathrm{MeV}$ for $Z=19$ and 35,55 and $75 \mathrm{MeV}$ for $Z=18$. Between each level, the variation of the multiplicity is $\Delta M=1$. Although statistics are not so good, the data at $13^{\circ}$ seem to show that these steps disappear at larger angles, indicating that they may be the signature of a rather direct process. This process could be the excitation of high energy states, deexciting in such a way that a large excitation energy variation corresponds to an almost constant angular momentum transfer. It would be an indication that for some correct energy and angular momentum matching, high $l$-pole giant resonances are excited. One should note that some of the observed steps approximately correspond to the structures observed in refs. [1] and [3]. But whereas it is often difficult to extract the bumps from the quasi-elastic or deep inelastic background, especially when only a $Z$ identification is performed, the multiplicity measurement allows a clean identification of phenomena correlated with a given value of the angular momentum. Even if differences in excitation energies and $Q$-values for the different isotopes smear the energy spectrum, they are not likely to wash out the multiplicity spectrum, since the $l$ values of the excited multipoles are smaller than the $l$ values associated with the background and not expected to depend very much on the $A$ of the fragment.

It is interesting to get an evaluation of the $l$-value of the possibly excited multipole. $\gamma$-rays are the ultimate stage of the deexcitation, following particle emission. In a system symmetric in the entrance channel and for a binary process, the centre of mass cross section should be symmetric around $Z=20$ if there were no evaporation from the fragments. Thus for any given excitation energy, our experiment allows the determination of the average number of charges $\Delta Z$ emitted in the deexcitation and of the variance $\sigma_{Z}$ of the $Z$ distribution. For the $N$ distribution, $\Delta N$ was evaluated from ref. [1] as well as from the ALICE code predictions [6], and included in the evaporation calculations. In our experiment, in the region of interest for collective excitations (40 MeV $\left.<E_{x}<75 \mathrm{MeV}\right) \Delta Z$ has been measured to vary linearly with the excitation energy between 1.2 and 2.4 , and $\sigma_{Z}$ between 1.5 and 3. $\Delta N$ has been taken as $\Delta Z /(2.3)$. This means that the steps observed in our experiments in $Z=18,19$ are the signature of excitations in the $Z=19,20$ or $Z=21$ initial fragments. Thus, the giant resonances, if such is the observed phenomenon, would be mainly excited in the ${ }^{40} \mathrm{Ca}$ itself, with the possibility of a subsequent particle transfer of one or two units (protons or neutrons). To evaluate the total angular momentum associated with the steps, particle emission from both fragments has to be taken in account. Since initial angular momenta involved are small.$\simeq 5 \hbar$ for each fragment), an evaluation of the angular momentum removed per emitted nucleon is between 0 and $1 \hbar$ [7]. $\gamma$ emissions carry away angular momenta between $1 \times M_{\gamma}$ (dipole emission only) and $1.8 \times M_{\gamma}$ (dipole plus quadrupole emission) [8]. With these assumptions, the total angular momentum windows corresponding to the steps in $Z=18$ are : $4 \hbar$ to $9 \hbar$ for the $35 \mathrm{MeV}$ step, $5 \hbar$ to $13 \hbar$ for the $55 \mathrm{MeV}$ step, $6 \hbar$ to $16 \hbar$ for the $75 \mathrm{MeV}$ step. Let us now consider how this total angular momentum is shared between the fragments. Two cases can be distinguished : a) In the direct process, only one $\mathrm{Ca}$ nucleus is excited in a giant resonance mode. Then the total angular momentum measured corresponds to the $l$-value of the excited multipole. The values of $l$ obtained in this hypothesis are very high but not unreasonable. For example Liu and Brown [9] have calculated that the isoscalar hexadecapole giant resonance in ${ }^{40} \mathrm{Ca}$ has a $l=4$ component at an excitation energy of $45 \mathrm{MeV}$ exhausting about $50 \%$ 
of the energy weighted sum rule; $b$ ) If both $\mathrm{Ca}$ nuclei are excited, then the $l$ windows corresponding to the steps are $2 \hbar-4.5 \hbar$ for $35 \mathrm{MeV}, 2.5 \hbar$ to $6.5 \hbar$ for $55 \mathrm{MeV}, 3 \hbar$ to $8 \hbar$ for $75 \mathrm{MeV}$. In this case, the energy of the resonances would be half the total excitation energies. It seems that the $l$-values extracted on the assumption of mutual excitation of the two $\mathrm{Ca}$ nuclei are more reasonable when compared to known experimental (isoscalar quadrupole resonance at $18 \mathrm{MeV}$ [10]) and theoretical (isoscalar octupole resonance at $33 \mathrm{MeV}$ [9]) evidence. This remains rather puzzling since such mutual excitation would be expected to be much less probable than single excitation. It is worth noting however that the extracted angular momenta are probably slightly overestimated, since they correspond to average multiplicities (including background multiplicity).

In conclusion, the variations of the multiplicity observed in this experiment support the hypothesis of the excitation of high-energy collective states during heavy-ion collisions, through a rather direct process. The measurement of the $\gamma$-ray multiplicity proved to be a sensitive tool for investigating details of the nuclear structure and variations of angular momentum at a relatively low excitation energy, where little particle evaporation takes place. Limits on the $l$ value of the excited multipole have been obtained. At higher excitation energies, where more particles are emitted, the $\gamma$-ray multiplicity is clearly not sufficient to determine the initial angular momentum [11] in such a low $A$ region of nuclei. As indicated above, in the course of the same experiment, more detailed information on particle emission has been obtained and will be published later.

Acknowledgments. - We would like to thank A. Winther and L. G. Moretto for continuous interest in this work and many fruitful discussions, M. Leblanc and Y. Sugiyama for valuable help during the experiment.

\section{References}

[1] Frascaria, N., Stéphan, C., Colombani, P., Garron, J. P., Jacmart, J. C., Riou, M. and Tassan-Got, L., Phys. Rev. Lett. 39 (1977) 918.

[2] Broglia, R. A., Dasso, C. H. and Winther, A., Phys. Lett. 61B (1976) 113.

BroGLiA, R. A., Lecture to the Nuclear Study Summer School, Dogashima, Aug. 28-Sept. 1 (1977) to be published.

[3] Frascaria, N. et al., Communication to the workshop on High Resolution Heavy Ion Physics at 20-100 MeV/A, Saclay, May 31-June 2 (1978), C. Volant, editor and to be published.

[4] Tricorre, H. et al., To be published.
[5] Broglia, R. A., Dasso, C. H., Pollarolo, G. and WinTHER, A., Phys. Rev. Lett. 41 (1978) 25.

[6] Plasil, F., Report ORNL/TM 6054.

[7] Williams, D. C. and Thomas, T. D., Nucl. Phys. A 92 (1967) 1

[8] Liotta, R. J. and Sorensen, R. A., Nucl. Phys. A 297 (1978) 136.

[9] Liu, K. F. and Brown, G. E., Nucl. Phys. A 265 (1976) 385.

[10] Bertrand, F. E., Ann. Rev. Nucl. Sci. 26 (1976) 457.

[11] Natowitz, J. B., Namboodiri, M. N., Kasiraj, P., EgGers, R., Adler, L., Gonthier, P., Cerruti, C. and Alleman, T., Phys. Rev. Lett. 40 (1978) 751. 